\title{
AVALIAÇÃO DO TEOR E DA ESTABILIDADE DE VITAMINAS DO COMPLEXO B E VITAMINA C EM BEBIDAS ISOTÔNICAS E ENERGÉTICAS
}

Fernanda J. de Castro, Rodrigo Scherer e Helena T. Godoy*

Departamento de Ciência de Alimentos, Faculdade de Engenharia de Alimentos, Universidade Estadual de Campinas, CP 6121, 13083-862 Campinas - SP, Brasil

Recebido em 13/5/05; aceito em 27/10/05; publicado na web em 24/3/06

\begin{abstract}
EVALUATION OF THE AMOUNT AND STABILITY OF B GROUP VITAMINS AND VITAMIN C IN POWER AND ISOTONIC BEVERAGES. Vitamin C stability and concentration was evaluated in isotonic beverages and $\mathrm{B}$ group vitamins $\left(\mathrm{B}_{1}, \mathrm{~B}_{2}, \mathrm{~B}_{3}, \mathrm{~B}_{5}\right.$ and $B_{6}$ ) in power beverages. The amount of vitamins was found to be above of that declared on the labels, even after the shelf life had been exceeded. A small decrease in the amount of B group vitamins was observed during the shelf life of the products. In the case of vitamin $\mathrm{C}$ this decrease was slightly higher. The present research shows the need of increased quality control and inspection.
\end{abstract}

Keywords: HPLC; pyridoxine; ascorbic acid.

\section{INTRODUÇÃO}

Bebidas isotônicas são repositores hidrolíticos formulados a partir da concentração variada de eletrólitos, associada a concentrações variadas de carboidratos, com o objetivo de reposição hídrica e eletrolítica decorrentes da prática de atividade física ${ }^{1}$. Esses produtos, segundo a ANVISA ${ }^{1}$ (Agência Nacional de Vigilância Sanitária), devem apresentar concentrações variadas de sódio, cloreto e carboidratos. Opcionalmente, podem conter potássio, vitaminas e/ou minerais em concentrações semelhantes às encontradas nos fluidos orgânicos. Por outro lado, os repositores energéticos são produtos formulados com nutrientes que permitam o alcance e/ou manutenção do nível apropriado de energia para atletas. São produtos nos quais os carboidratos devem constituir, no mínimo, $90 \%$ dos nutrientes energéticos presentes na formulação. Opcionalmente, estes produtos podem conter vitaminas e/ou minerais ${ }^{1}$, ou ainda cafeína e alguns aminoácidos.

A fortificação de bebidas isotônicas e energéticas com vitaminas tem sido uma prática adotada pela maioria das indústrias do ramo, sendo as vitaminas do complexo $\mathrm{B}$, vitaminas $\mathrm{C}$ e ácido fólico as principais encontradas nos produtos. Vitaminas lipossolúveis, como A e E, também podem ser encontradas pelo uso de microencapsulação. Além da função repositória após exercícios físicos, as bebidas fortificadas podem ser utilizadas para outros objetivos, como por ex., para controlar deficiências vitamínicas em comunidades carentes, além de prevenir doenças.

Um estudo realizado no sul da Espanha revelou que a população local consome de 2 a 3 vezes abaixo dos níveis recomendados para as vitaminas $\mathrm{B}_{6}, \mathrm{~B}_{12}$ e folatos ${ }^{2}$. Abrams et al. ${ }^{3}$ avaliaram a eficácia de bebidas fortificadas com vitaminas em crianças de 6 a 11 anos em relação os níveis séricos de vitaminas, durante 8 semanas. Os autores citam que os níveis séricos de riboflavina e ácido fólico aumentaram significativamente e, ainda, concluem que a fortificação de bebidas para populações de risco pode ser uma alternativa viável para controle de deficiência vitamínica, devido à fácil aceitação do produto. Sichert-Hellert e Kersting ${ }^{4}$ relatam que o consumo de vitaminas $\mathrm{B}_{1}, \mathrm{~B}_{2}, \mathrm{~B}_{3}, \mathrm{~B}_{6}, \mathrm{~A}, \mathrm{E}, \mathrm{C}$ e ácido fólico aumentou entre jovens e adolescentes na Alemanha devido ao consu-

*e-mail: helena@fea.unicamp.br mo de bebidas fortificadas, como isotônicos e energéticos. Rimm et al. ${ }^{5}$ relatam que o risco de doenças coronárias foi reduzido em mulheres com consumo regular de vitamina $\mathrm{B}_{6} \mathrm{e}$ ácido fólico. Zhang et $a l .{ }^{6}$ relatam uma associação inversa altamente significativa entre os níveis plasmáticos de folatos e vitamina $\mathrm{B}_{12}$ com o risco de câncer de mama. No Brasil, a extensão e a freqüência do consumo de produtos vitamínicos ainda são praticamente desconhecidas, embora haja registro de aumento da importação e das vendas desses produtos.

Além dos trabalhos citados acima, encontram-se na literatura inúmeras outras aplicações de bebidas repositoras hidrolíticas ou energéticas, como o uso em atletas idosos ${ }^{7}$; o consumo de vitamina $\mathrm{B}_{2}$ aumenta os níveis de cálcio e magnésio no fígado de ratos $^{8}$; prevenção de acidentes em motoristas que dirigem à noite pelo aumento da atenção e redução do sono $^{9}$; efeito positivo sobre a performance mental aumentando a capacidade do processamento da informação $0^{10-12}$, entre outras.

Para garantir a segurança do consumidor que procura essas bebidas, é necessário que o nível dos micronutrientes obedeça a ingestão diária recomendada (IDA), além de estar em acordo com o declarado no rótulo pelos fabricantes. A literatura apresenta métodos analíticos para determinação das vitaminas nesses produtos como, por ex., a determinação das vitaminas do complexo B pode ser realizada através de métodos microbiológicos ${ }^{13,14}, \mathrm{CLAE}^{15-22}$, cromatografia micelar eletrocinética ${ }^{23}$, cromatografia gasosa ${ }^{24}$ e por espectrofotometria, empregando sistema de análises por injeção em fluxo ${ }^{25}$. Entretanto, a literatura é deficiente em relação à avaliação dos níveis e da estabilidade das vitaminas em bebidas fortificadas. Portanto, o objetivo deste trabalho é avaliar o teor e a estabilidade da vitamina $\mathrm{C}$ e das vitaminas do complexo $\mathrm{B}$ em bebidas isotônicas e energéticas disponíveis comercialmente.

\section{PARTE EXPERIMENTAL}

Foram adquiridas 14 bebidas isotônicas líquidas de 3 fabricantes diferentes, codificados por SSL, GLN e CRF, divididas em 7 sabores e 3 tipos de embalagens diferentes. Durante a execução deste experimento, o fabricante GLN suspendeu e relançou o mesmo produto com redução do teor de vitamina $\mathrm{C}$ e, para diferenciar, o produto relançado foi codificado como GLNN. Foram adquiridas 
também, 5 marcas diferentes de energéticos, codificados por WRP, OMD, LAT, YLH e DBB. As bebidas foram adquiridas em supermercados e farmácias da região de Campinas, São Paulo. Todas as amostras foram analisadas em três diferentes lotes, sendo que cada lote foi formado pela homogeneização do conteúdo total de duas embalagens, dando-se preferência a embalagens com data de fabricação recente. As amostras foram analisadas dentro do prazo de validade do produto estabelecido pelo fabricante. Em decorrência da abertura das embalagens e do tempo de análise, as análises foram conduzidas sempre em duplicata. Os padrões das vitaminas B (mononitrato de tiamina, riboflavina, hidrocloridrato de piridoxina, ácido nicotínico, nicotinamida e pantatenato de cálcio) foram cedidos pela F. Hoffman-La Roche. O padrão de ácido ascórbico utilizado foi da Sigma.

A vitamina $C$ foi determinada pelo método oficial titulométrico $\mathrm{AOAC}^{26}$. As vitaminas $\mathrm{B}_{1}$ (tiamina), $\mathrm{B}_{2}$ (riboflavina), $\mathrm{B}_{3}$ (ácido nicotínico), $\mathrm{B}_{5}$ (ácido pantotênico) e $\mathrm{B}_{6}$ (piridoxina) foram determinadas pela metodologia desenvolvida por nosso laboratório, utilizando $\mathrm{CLAE}^{18}$. O sistema cromatográfico usado consistiu em um cromatógrafo Varian equipado com detector de arranjo de diodos modelo 9065 e de fluorescência modelo HP 1046A, bomba modelo 9010 e alça de amostragem com $20 \mu \mathrm{L}$ de injeção. Foi utilizada uma coluna Spherisorb $C_{18}$ ODS-2 $(150$ x 46 mm, $5 \mu \mathrm{m})$ (SigmaAldrich) com coluna de guarda $\mathrm{C}_{18}(5 \mu \mathrm{m})$ (Varian). A fase móvel para separação das vitaminas consistiu em acetonitrila, solução aquosa ( $5 \mathrm{mM}$ ácido hexasulfônico e $0,15 \%$ trietilamina ajustado para $\mathrm{pH} 2,8$ com $\mathrm{H}_{2} \mathrm{SO}_{4}$ ) e metanol com fluxo de $0,7 \mathrm{~mL} / \mathrm{min}$, através de um gradiente inicial de $2 \%$ acetonitrila e $98 \%$ solução aquosa até 3 e $97 \%$, respectivamente, em 3 min, seguido de um gradiente linear de $2 \%$ de acetonitrila, $41 \%$ de solução aquosa e $57 \%$ de metanol até $20 \mathrm{~min}$. O equilíbrio da coluna para as condições iniciais foi realizado em $20 \mathrm{~min}$. As vitaminas foram monitoradas em $254 \mathrm{~nm}$ até $9 \mathrm{~min}, 278 \mathrm{~nm}$ de 9 até $15 \mathrm{~min}$ e 254 a partir de $15 \mathrm{~min}$. Nas amostras de bebidas, apesar de serem matrizes diferentes das avaliadas por Agostini e Godoy ${ }^{18}$, em virtude de sua simples composição, estas foram apenas filtradas (HAWP 0013, 0,45 $\mu \mathrm{m}$ Millipore) e injetadas diretamente no cromatógrafo, não apresentando problemas com interferentes. A identificação foi feita por comparação dos tempos de retenção, espectros de absorção UV/ visível obtidos no detector de arranjo de diodos e de fluorescência, além da co-cromatografia.

\section{RESULTADOS E DISCUSÃO}

A Tabela 1 mostra o teor de vitamina $\mathrm{C}$ das 14 diferentes bebidas isotônicas analisadas em três diferentes lotes. Para todos os fabricantes, independente do tipo de embalagem ou vida de prateleira, o valor encontrado foi superior ao declarado. No caso do fabricante $\mathrm{CRF}$, a quantidade de vitamina $\mathrm{C}$ encontrada foi superior a 10 vezes a quantidade declarada no rótulo, enquanto que na marca GLN o resultado encontrado foi $25 \%$ maior que o declarado na embalagem. Valores tão elevados podem ser devidos à sobredosagem pois, tanto os fornecedores de premixes sugerem uma sobredosagem para compensar perdas durante o processamento, como as empresas em adicionarem quantidades maiores de vitaminas para garantir os níveis até o prazo de validade do produto. Su et al. ${ }^{27}$ avaliaram o teor de vitamina $\mathrm{C}, \mathrm{B}_{1}$ e $\mathrm{B}_{2}$ em 30 alimentos para recém-nascidos. Os resultados mostraram que os valores encontrados para as 3 vitaminas, na maioria dos alimentos, foram maiores que as quantidades declaradas no rótulo, chegando a $250 \%$ em algumas amostras.

Segundo a resolução RDC no $360 / 03$ da ANVISA ${ }^{28}$, item 3.5.1. do Regulamento Técnico Sobre Rotulagem Nutricional de Ali- mentos Embalados, a variação máxima dos nutrientes declarados no rótulo permitida é de $20 \%$ a mais que o declarado pelo fabricante. Sendo assim, a marca GLN apresentou melhores resultados, entretanto, os demais fabricantes não estão de acordo com a legislação brasileira, devendo rever o processo de fortificação das bebidas.

Sabendo-se que a ingestão diária recomendável (IDR) para vitamina C é de $60 \mathrm{mg}$, uma dose (garrafa de $400 \mathrm{~mL}$ ) de qualquer marca avaliada ultrapassa o nível recomendado. Além disso, a marca GLN pode contribuir com $400 \mathrm{mg}$ de vitamina $\mathrm{C}$, ou seja, ingerindo apenas um isotônico ao dia, não considerando o restante da alimentação, o indivíduo consumirá mais de 6 vezes a quantidade recomendável desta vitamina. Doses diárias entre 2 e 10 g de vitamina $\mathrm{C}$ podem causar náuseas, vômitos, diarréias e cálculo renal ${ }^{29}$.

Foram analisados, também, os teores das vitaminas $\mathrm{B}_{1}, \mathrm{~B}_{2}, \mathrm{~B}_{6}$ ácido nicotínico e ácido pantotênico em 5 bebidas estimulantes de fabricantes diferentes, 3 delas em latas (YLH, DBB, WRP) e 2 em embalagem tipo PET (OMD, LAT) como mostra a Tabela 2. Não foi verificada a presença de interferentes devido a simples composição das bebidas, além disso, os coeficientes de variação obtidos entre as determinações em duplicata não ultrapassaram 6\%. Notase que, com exceção do ácido pantotênico e da vitamina $B_{1}$, as quantidades das vitaminas presentes são superiores às descritas nos rótulos. No caso do ácido nicotínico, foi encontrada uma quantidade de aproximadamente $55 \%$ a maior que a declarada em todas as marcas, enquanto que para a vitamina $\mathrm{B}_{6}$ foram encontrados valores de 50 a $200 \%$ a mais que o especificado no rótulo. Entretanto, a marca DBB apresentou 34\% a menos em relação ao declarado para a vitamina $B_{6}$. No caso da vitamina $B_{2}$, os teores encontrados nas marcas WRP, YLH e DBB foram de 10 a 15 vezes maiores que a quantidade declarada. A quantidade de ácido pantotênico presente representou apenas cerca de $10 \%$ do valor declarado para todos os fabricantes. A vitamina $\mathrm{B}_{1}$ foi encontrada somente na marca DBB, representando apenas cerca de $30 \%$ do conteúdo especificado no rótulo. Considerando a resolução da $\mathrm{RDC} \mathrm{n}^{\circ} 360 / 03$ da $\mathrm{ANVISA}^{28}$, citada anteriormente, nenhum dos fabricantes avaliados está de acordo com a legislação.

Segundo a ANVISA ${ }^{1}$, a IDR, para as vitaminas $\mathrm{B}_{1}, \mathrm{~B}_{2}, \mathrm{~B}_{6}, \mathrm{~B}_{5} \mathrm{e}$ $\mathrm{B}_{3}$ são, respectivamente, 1,4, 1,6, 2,0, 6,0 e $18 \mathrm{mg}$, para adultos. Considerando-se uma embalagem de $400 \mathrm{~mL}$ do fabricante DBB, o teor de vitamina $B_{2}$ pode chegar a $57 \mathrm{mg}$, ou seja, uma quantidade 35 vezes superior ao recomendado pela ANVISA ${ }^{1}$. Para os fabricantes OMD e LAT, os teores de vitamina $\mathrm{B}_{6}$ chegam a atingir 6,2 vezes a IDR, considerando-se uma embalagem de $400 \mathrm{~mL}$. A quantidade de vitamina $\mathrm{B}_{3}$ encontrada em todas as marcas foi semelhante, sendo 2,6 vezes maior que a IDR, ao se considerar uma embalagem de $400 \mathrm{~mL}$. A quantidade de vitamina $\mathrm{B}_{5}$ está dentro das recomendações da ANVISA ${ }^{1}$ em todas as amostras (Tabela 2).

Doses acima de $3 \mathrm{~g}$ ao dia de ácido nicotínico $\left(\mathrm{B}_{3}\right)$ podem resultar em úlceras pépticas ${ }^{30}$. Alterações cardiovasculares e vasodilatação podem estar associadas ao consumo de doses elevadas de tiamina $\left(\mathrm{B}_{1}\right)$ e megadoses de piridoxina $\left(\mathrm{B}_{6}\right)$ podem resultar em neuropatias periféricas prolongadas ${ }^{29}$.

Lin $e t a l .{ }^{24}$, ao avaliarem o teor de nicotinamida em energéticos, encontraram uma quantidade de 3 a 5 vezes acima dos níveis diários recomendados. Albalá-Hurtado et al. ${ }^{31}$ encontraram níveis ao redor de $50 \%$ acima para as vitaminas nicotinamida e piridoxina em leites formulados para crianças. Os trabalhos acima citados, assim como o presente trabalho, revelam que as informações declaradas nos rótulos devem ser revistas, assim como o controle de qualidade dos fabricantes e a ação dos órgãos fiscalizadores, para melhor segurança dos consumidores. 
Tabela 1. Teores de vitamina C (ácido ascórbico) em bebidas isotônicas

\begin{tabular}{|c|c|c|c|c|c|c|c|}
\hline \multirow[t]{2}{*}{ Marca } & \multirow[t]{2}{*}{ Sabor/lote } & \multirow[t]{2}{*}{ Embalagem } & \multirow{2}{*}{$\begin{array}{c}\text { Vida de } \\
\text { Prateleira* } \\
\text { (meses) }\end{array}$} & \multirow[t]{2}{*}{$\begin{array}{l}\text { Teor de vit. } \mathrm{C}^{*} \\
(\mathrm{mg} \text { por } 100 \mathrm{~mL})\end{array}$} & \multicolumn{3}{|c|}{$\begin{array}{l}\text { Ácido ascórbico } \\
\text { (mg por } 100 \mathrm{~mL})\end{array}$} \\
\hline & & & & & M & $\mathrm{SD}$ & $\mathrm{CV}(\%)$ \\
\hline \multirow[t]{9}{*}{ SSL } & uva-limão/1 & vidro & 6 & 4,8 & 22,7 & 0,4 & 1,7 \\
\hline & 12 & & 6 & 4,8 & 24,1 & 0,0 & 0,0 \\
\hline & 13 & & 6 & 4,8 & 23,4 & 0,3 & 1,2 \\
\hline & mix-tropical/1 & vidro & 6 & 4,8 & 22,6 & 0,3 & 1,3 \\
\hline & 12 & & 6 & 4,8 & 16,9 & 0,3 & 1,7 \\
\hline & 13 & & 6 & 4,8 & 21,7 & 0,1 & 0,4 \\
\hline & Guaraná/1 & vidro & 6 & 4,8 & 27,7 & 1,0 & 3,6 \\
\hline & 12 & & 6 & 4,8 & 24,9 & 0,3 & 1,2 \\
\hline & 13 & & 6 & 4,8 & 25,5 & 0,4 & 1,5 \\
\hline \multirow[t]{9}{*}{$\mathrm{CRF}$} & Laranja/1 & PET & 12 & 9 & 95,8 & 0,8 & 0,8 \\
\hline & 12 & & 12 & 9 & 96,3 & 0,5 & 0,5 \\
\hline & 13 & & 12 & 9 & 92,1 & 0,2 & 0,2 \\
\hline & Cupuaçu/1 & PET & 12 & 9 & 96,3 & 0,0 & 0,0 \\
\hline & 12 & & 12 & 9 & 97,6 & 0,2 & 0,2 \\
\hline & 13 & & 12 & 9 & 99,1 & 0,2 & 0,2 \\
\hline & Maracujá/1 & PET & 12 & 9 & 87,4 & 0,1 & 0,1 \\
\hline & 12 & & 12 & 9 & 95,3 & 0,4 & 0,4 \\
\hline & 13 & & 12 & 9 & 93,8 & 0,3 & 0,3 \\
\hline \multirow[t]{18}{*}{ GLN } & Cupuaçu/1 & tetrapak & 12 & 60 & 90,9 & 1,0 & 1,1 \\
\hline & 12 & & 12 & 60 & 92,2 & 2,0 & 2,1 \\
\hline & 13 & & 12 & 60 & 113,4 & 3,1 & 2,7 \\
\hline & Cupuaçu/1 & PET & 12 & 60 & 89,2 & 0,3 & 0,3 \\
\hline & 12 & & 12 & 60 & 86,3 & 1,0 & 1,1 \\
\hline & 13 & & 12 & 60 & 97,4 & 0,6 & 0,6 \\
\hline & Laranja/1 & tetrapak & 12 & 60 & 93,2 & 6,0 & 6,4 \\
\hline & $/ 2$ & & 12 & 60 & 87,8 & 1,0 & 1,1 \\
\hline & 13 & & 12 & 60 & 97,5 & 5,3 & 5,4 \\
\hline & Acerola/1 & tetrapak & 12 & 60 & 88,7 & 0,7 & 0,7 \\
\hline & 12 & & 12 & 60 & 87,6 & 2,0 & 2,2 \\
\hline & 13 & & 12 & 60 & 93,6 & 1,8 & 1,9 \\
\hline & Acerola/1 & PET & 12 & 60 & 96,9 & 1,0 & 1,0 \\
\hline & 12 & & 12 & 60 & 93,8 & 1,0 & 1,0 \\
\hline & 13 & & 12 & 60 & 89,7 & 0,8 & 0,8 \\
\hline & Maracujá/1 & PET & 12 & 60 & 102,2 & 0,7 & 0,7 \\
\hline & 12 & & 12 & 60 & 98,0 & 7,0 & 7,0 \\
\hline & 13 & & 12 & 60 & 100,6 & 3,8 & 3,7 \\
\hline \multirow[t]{12}{*}{ GLNN } & Laranja/1 & tetrapak & 12 & 9 & 71,4 & 0,9 & 1,2 \\
\hline & 12 & & 12 & 9 & 86,2 & 0,4 & 0,4 \\
\hline & 13 & & 12 & 9 & 79,5 & 0,5 & 0,6 \\
\hline & Maracujá/1 & tetrapak & 12 & 9 & 64,9 & 0,9 & 1,3 \\
\hline & 12 & & 12 & 9 & 77,3 & 0,3 & 0,3 \\
\hline & 13 & & 12 & 9 & 69,8 & 0,3 & 0,4 \\
\hline & Cupuaçu/1 & tetrapak & 12 & 9 & 55,3 & 0,6 & 1,0 \\
\hline & $/ 2$ & & 12 & 9 & 51,7 & 0,5 & 0,9 \\
\hline & 13 & & 12 & 9 & 64,8 & 0,6 & 0,9 \\
\hline & Acerola/1 & tetrapak & 12 & 9 & 69,8 & 0,3 & 0,4 \\
\hline & $/ 2$ & & 12 & 9 & 76,3 & 0,2 & 0,2 \\
\hline & 13 & & 12 & 9 & 77,8 & 0,2 & 0,2 \\
\hline
\end{tabular}

M: média; SD: desvio padrão; CV: coeficiente de variação. * Declarado pelo fabricante. 
Tabela 2. Teores de vitaminas do complexo B em bebidas energéticas

\begin{tabular}{|c|c|c|c|c|c|}
\hline \multirow[t]{2}{*}{ Amostras/Lote } & \multicolumn{5}{|c|}{ Vitaminas $(\mathrm{mg} / 100 \mathrm{~mL})^{*}$} \\
\hline & $\begin{array}{c}\text { Ác. } \\
\text { Pantotênico }\end{array}$ & $\begin{array}{c}\text { Ác. } \\
\text { Nicotínico }\end{array}$ & $\mathrm{B}_{1}$ & $\mathrm{~B}_{2}$ & $\mathrm{~B}_{6}$ \\
\hline WRP-1 & 0,10 & 10,10 & 0,00 & 6,90 & 1,1 \\
\hline WRP-2 & 0,12 & 11,22 & 0,00 & 7,51 & 1,2 \\
\hline WRP-3 & 0,10 & 10,97 & 0,00 & 6,57 & 1,1 \\
\hline $\begin{array}{l}\text { Rótulo** } \\
\text { (mg/100 mL) }\end{array}$ & 2,00 & 7,20 & 0,00 & 0,60 & 0,8 \\
\hline OMD-1 & 0,20 & 11,10 & 0,00 & 0,00 & 3,2 \\
\hline OMD-2 & 0,22 & 11,34 & 0,00 & 0,00 & 2,9 \\
\hline OMD-3 & 0,20 & 10,98 & 0,00 & 0,00 & 3,1 \\
\hline $\begin{array}{l}\text { Rótulo** } \\
\text { (mg/100 mL) }\end{array}$ & 2,00 & 8,00 & 0,00 & 0,00 & 1,0 \\
\hline LAT-1 & 0,22 & 11,54 & 0,00 & 0,00 & 2,8 \\
\hline LAT-2 & 0,18 & 12,08 & 0,00 & 0,00 & 2,8 \\
\hline LAT-3 & 0,20 & 12,50 & 0,00 & 0,00 & 3,1 \\
\hline $\begin{array}{l}\text { Rótulo** } \\
\text { (mg/100 mL) }\end{array}$ & 2,00 & 8,00 & 0,00 & 0,00 & 1,0 \\
\hline YLH-1 & 0,20 & 10,80 & 0,00 & 6,90 & 1,2 \\
\hline YLH-2 & 0,25 & 11,14 & 0,00 & 6,56 & 1,1 \\
\hline YLH-3 & 0,22 & 11,20 & 0,00 & 7,01 & 1,2 \\
\hline $\begin{array}{l}\text { Rótulo** } \\
\text { (mg/100 mL) }\end{array}$ & 2,00 & 7,00 & 0,00 & 0,60 & 0,8 \\
\hline DBB-1 & 0,20 & 13,30 & 0,20 & 14,30 & 0,7 \\
\hline DBB-2 & 0,23 & 12,12 & 0,26 & 13,11 & 0,6 \\
\hline DBB-3 & 0,21 & 12,65 & 0,34 & 14,05 & 0,7 \\
\hline $\begin{array}{l}\text { Rótulo** } \\
(\mathrm{mg} / 100 \mathrm{~mL})\end{array}$ & 3,00 & 9,00 & 0,70 & 0,80 & 1,0 \\
\hline
\end{tabular}

*Resultados são a média de determinações em duplicata com coeficientes de variação inferiores a $6 \%$; **dados fornecidos pelo fabricante.
A Tabela 3 mostra os resultados referentes à estabilidade da vitamina $\mathrm{C}$ durante a vida de prateleira das bebidas isotônicas. Neste estudo foram escolhidas três amostras dos fabricantes SSL e GLN. Ao final do prazo de validade dos produtos da marca SSL, a concentração da vitamina $C$ nos sabores uva/limão e mix tropical foi reduzida em aproximadamente $25 \%$, entretanto os teores de vitamina $\mathrm{C}$ ainda se encontravam muito acima do declarado (350\%). Não foi possível encontrar no mercado amostras da marca SSL do sabor guaraná com data de fabricação recente, portanto, esse produto só pode ser analisado de 4 a 6 meses após a fabricação. Entretanto, a exemplo dos outros dois sabores produzidos pelo mesmo fabricante, os valores de vitamina $C$ representavam cerca de 5 vezes o teor descrito no rótulo, ao final da validade do produto.

Nos produtos da marca GLN, mesmo após 12 meses de fabricação, o teor de vitamina $\mathrm{C}$ encontrado foi cerca de $50 \%$ superior ao declarado no rótulo, entretanto se manteve praticamente constante, com perdas na ordem de $5 \%$ ao longo da vida útil. Gimenez et $a .^{32}$ avaliaram a estabilidade da vitamina $\mathrm{C}$ em suplementos comerciais (ampolas). Ao final do prazo de validade, houve uma perda de 10,4-19,3\%.

Assim como os isotônicos, as bebidas energéticas YLH, WRP, OMD e LAT adquiridas foram armazenadas no laboratório em condições semelhantes às encontradas na maioria dos supermercados e farmácias, em termos de luminosidade e temperatura, para avaliação da estabilidade das vitaminas do complexo B durante a vida útil das bebidas. Os resultados estão apresentados na Tabela 4. As marcas OMD e LAT tiveram uma redução drástica na quantidade de ácido pantotênico após 4 meses, sendo essas envasadas em embalagens tipo PET, enquanto que as marcas WRP e YLH, envasadas em latas, tiveram as quantidades de ácido pantotênico mantidas praticamente constantes. $\mathrm{O}$ ácido nicotínico, as vitaminas $\mathrm{B}_{2}$ e $\mathrm{B}_{6}$ tiveram poucas alterações, com perda ao redor de $15 \%$ ao longo de 4 meses em todas as marcas avaliadas; apenas na marca YLH houve uma redução de $40 \%$ no teor de vitamina $\mathrm{B}_{6}$.

Tabela 3. Avaliação da estabilidade da vitamina C (ácido ascórbico) durante a vida de prateleira

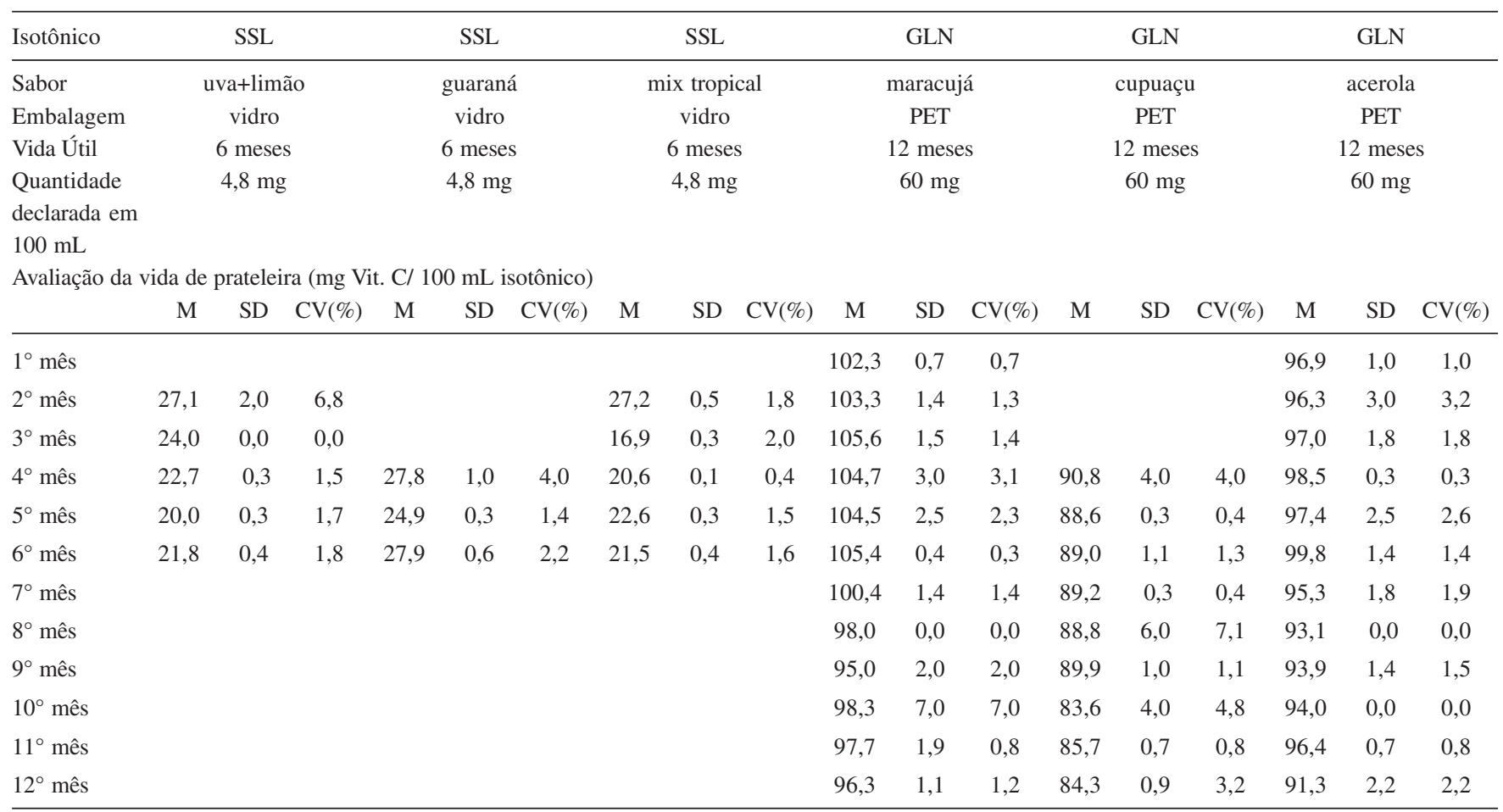

M: média; SD: desvio padrão; $\mathrm{CV}$ : coeficiente de variação 
Tabela 4. Avaliação da estabilidade de vitaminas do complexo B em bebidas energéticas

\begin{tabular}{lcccc}
\hline $\begin{array}{l}\text { Amostras/dias } \\
\text { após a } \\
\text { fabricação }\end{array}$ & $\begin{array}{c}\text { Ác. } \\
\text { Pantotênico }\end{array}$ & $\begin{array}{c}\text { Ác. } \\
\text { Nicotínico }\end{array}$ & $\mathrm{B}_{2}$ & $\mathrm{~B}_{6}$ \\
\hline WRP-15 & 0,10 & 10,10 & 6,90 & 1,10 \\
WRP-60 & 0,08 & 9,32 & 7,12 & 0,90 \\
WRP-118 & 0,07 & 9,00 & 6,32 & 0,82 \\
& & & & \\
OMD-20 & 0,20 & 11,10 & 0,00 & 3,20 \\
OMD-60 & 0,11 & 11,14 & 0,00 & 2,67 \\
OMD-122 & 0,05 & 10,28 & 0,00 & 2,71 \\
& & & & \\
LAT-20 & 0,18 & 11,54 & 0,00 & 2,86 \\
LAT-62 & 0,08 & 11,02 & 0,00 & 2,63 \\
LAT-120 & 0,03 & 10,70 & 0,00 & 2,54 \\
& & & & \\
YLH-10 & 0,20 & 10,80 & 6,90 & 1,20 \\
YLH-59 & 0,18 & 9,35 & 6,14 & 1,02 \\
YLH-120 & 0,16 & 8,87 & 5,81 & 0,74 \\
\hline
\end{tabular}

* Resultados são a média de determinações em duplicata; coeficientes de variação inferiores a $6 \%$

\section{CONCLUSÕES}

As quantidades de vitamina $\mathrm{C}$ encontradas nos repositores hidrolíticos avaliados estavam acima das quantidades declaradas pelos fabricantes, chegando a atingir 10 vezes o teor declarado no rótulo. Para as vitaminas do complexo $\mathrm{B}$, os valores encontrados variaram cerca de 10 vezes a menos e 15 vezes a mais que o declarado no rótulo. A quantidade de vitamina $\mathrm{B}_{2}$ encontrada chegou a ser 35 vezes superior à ingestão diária recomendada. Houve uma perda de vitamina $\mathrm{C}$ durante a vida útil dos isotônicos avaliados na ordem de 30 e $5 \%$ nas duas marcas avaliadas. As vitaminas do complexo B tiveram poucas alterações ao longo da vida útil, entretanto, o ácido pantotênico foi drasticamente reduzido nos energéticos envasados em garrafas PET. Portanto, ao verificar esses elevados níveis de discordância entre os reais teores de vitaminas e quantidades declaradas no rótulo pelos fabricantes, nota-se a necessidade de aumentar o controle de qualidade sobre esses produtos.

\section{AGRADECIMENTOS}

À FAPESP e à FINEP-RECOPE pelo auxílio à pesquisa.

\section{REFERÊNCIAS}

1. ANVISA, 1998; Portaria no 222, de 24 de março de 1998, http:// www.anvisa.gov.br/alimentos/legis/especifica/regutec.htm, acessada em Março 2005

2. Planells, E.; Sanchez, C.; Montellano, M. A.; Mataix, J.; Llopis, J.; Eur. J. Clin. Nutr. 2003, 57, 777.

3. Abrams, S. A.; Mush, A.; Hilmers, D. C.; Griffin, I. J.; Davila, P.; Allen, L.; J. Nutr, 2003, 133, 1834

4. Sichert-Hellert, W.; Kersting, M.; Int. J. Vitam. Nutr. Res. 2001, 71, 356.

5. Rimm, E. B.; Willett, W. C.; Hu, F. B.; Sampson, L.; Colditz, G. A.; Manson, J. E.; Hennekens, C.; Stampfer, M. J.; JAMA, J. Am. Med. Assn. 1998, 279, 359.

6. Zhang, S. M.; Willett, W. C.; Selhub, J.; Hunter, D. J.; Giovannucci, E. L.; Holmes, M. D.; Colditz, G. A.; Hankinson, S. E.; J. Nat. Can. Inst. 2003, 95, 373

7. Campbell, W. W.; Geik, R. A.; Nutrition 2004, 20, 603.

8. Gralak, M. A.; Bertrandt, J.; Klos, A.; Stryczek, A. B.; Piastowska, A. W.; Morka, A.; Debski, B.; Trace Elem. Electroly. 2004, 21, 89.

9. Reyner, L. A.; Horne, J. A.; Phys. Behav. 2002, 75, 331.

10. Seidl, R.; Peyrl, A.; Nicham, R.; Hauser, E.; Amino Acids 2000, 19, 635.

11. Warburton, D. M.; Bersellini, E.; Sweeney, E.; Psychopharmacology 2001, $158,322$.

12. Scholey, A. B.; Kennedy, D. O.; Psychopharmacology 2004, 176, 320.

13. Tanner, J. T.; Smith, J.; Defibaugh, P.; Angyal, G.; Villalobos, M.; Bueno, M. P.; McGarrahan, E.; Wehr, H. M.; Muniz, J. F.; Hollis, B. W.; Koh, Y.; Reich, P.; Simpson, K. L.; J. AOAC Int. 1988, 71, 607.

14. Premaratne, R. J.; Cousin, M. A.; J. Dairy Sci. 1991,74, 788.

15. Chase, G. W.; Landen, W. O.; Eiternmiller, R. R.; Soliman, A. G.; J. AOAC Int. 1992, 75, 561 .

16. Muñoz, A.; Ortiz, A.; Murcia, M. A.; Food Chem. 1994, 49, 203.

17. Arella, F.; Lahely, S.; Bourguignon, J. B.; Hasselmann, C.; Food Chem. 1996, 56,81 .

18. Agostini, T. S.; Godoy, H. T.; J. High Resol. Chromatogr. 1997, 20, 245.

19. Ollilainen, V.; Finglas, P. M.; van den Berg, H.; Froidmont-Gortz, I.; J. Agric. Food. Chem. 2001, 49, 315.

20. Woollard, D. C;. Indyk, H. E.; J. AOAC Int. 2002, 85, 945.

21. Vinas, P.; Balsalobre, N.; Lopez-Erroz, C.; Hernandez-Cordoba, M.; J. Agric. Food. Chem. 2004, 52, 1789.

22. Carver, J. D.; Am. J. Clin. Nutr. 2003, 77, 1550

23. Okamoto, H.; Nakajima, T.; Ito. Y.; J. Pharmaceut. Biomed. 2003, $30,815$.

24. Lin, H. J.; Chen, C. W.; Hwang, B. S.; Choong, Y. M.; J. Food Drug Anal. 2000, 8,113 .

25. Aniceto, C.; Canaes, L. S.; Fatibello-Filho, O.; Cavalheiro, C. C. S.; Quim. Nova 2000, 23, 637.

26. Cuniff, P.; Official Methods of Analysis of AOAC International, $17^{\text {th }}$ ed., AOAC International, Ganithersburg, Maryland, 1997.

27. Su, S. C.; Lee, S. C.; Wong, Y. H.; Chou, S. S.; J. Chin. Nutrit. Soc. 1995, 20, 157.

28. ANVISA, 2003; Resolução RDC n 360 de 23 de dezembro de 2003, http:/ /www.anvisa.gov.br/alimentos/legis/especifica/rotuali.htm, acessada em Julho 2005.

29. Podmore, I. D.; Griffiths, H. R.; Herbert, K. E.; Mistry, N.; Mistry, P.; Lunec, J.; Nature 1998, 392, 559

30. Bloch, A. S.; Nutrition 2000, 16, 236

31. Albala-Hurtado, S.; Veciana-Nogues, M. T.; Riera-Valls, E.; Marine-Font, A.; Vidal-Carou, M. C.; J. Dairy Res. 2000, 67, 225.

32. Gimenéz, R.; Cabrera, C.; Ollala, M.; Ruiz, M. D.; Lopes, M. C.; Int. J. Food Sci. Nutrit. 2002, 53, 509. 